\title{
From the quarantine diary of an emergency physician: the coronavirus and the dysautonomic storm
}

\author{
Simone Vanni ${ }^{1}\left[\right.$ Peiman Nazerian ${ }^{2} \cdot$ Giancarlo Bini $^{3}$
}

Received: 31 March 2020 / Accepted: 18 April 2020 / Published online: 9 May 2020

(c) Società Italiana di Medicina Interna (SIMI) 2020

The preparations are fervent, the organizational changes are many and rapidly evolving, meetings follow one another. I go up and down the stairs with my mask and I seem to be struggling more than usual, is it the mask? When I talk and I get excited about a subject I feel like I have to finish the sentence first, is it the tension?

I spend $12 \mathrm{~h}$ in the hospital, in the evening at home I find G. (15 years old) who has fever and diarrhea. I notice that the other two have an insistent dry cough; B. (54) has a sore throat...

Saturday morning (March 14th) I wake up exhausted, but I have to go to work... The road in the car has less traffic than usual. The sunny morning seems promising, but my strength is lacking, I need to get home early. I have a feeling. I tell my family that many colleagues have gone to sleep on their own and I get the kids to prepare a bed in the study and I lie down and try to sleep. I can't, even though I feel very tired. I stay in bed, it's snack time, you hear the news about the pandemic, the tension rises. I feel I have a temperature, a feeling of warmth in my face with cold extremities, but no shivers. I take my temperature repeatedly, $36.1-36.2^{\circ} \mathrm{C}$. Is it my impression? Nonetheless, my face is hot, and I am tachycardic (90-100/min, usually 60-70). I have an oxymeter at home, $98 \%$. After dinner, I try to sleep but I can't, a polyuria starts. Clear, abundant urine, one, two, three times. Maybe it's anxiety? During the night around one o'clock I finally get a high-temperature $\left(38-39^{\circ} \mathrm{C}\right)$; this is, at last, an objective sign of my malaise. Tachycardia $120 / \mathrm{min}$. I stay in bed, I have a headache and cervical pain as if from meningism.

Simone Vanni

simone2.vanni@uslcentro.toscana.it

1 Emergency Medicine Unit, San Giuseppe Hospital, Azienda USL Toscana Centro, Empoli, Italy

2 Emergency Medicine Department, Azienda Ospealiero Universitaria Careggi, Firenze, Italy

3 Internal Medicine Unit, Santo Stefano Hospital, Azienda USL Toscana Centro, Prato, Italy
Even Lasegue's sign also seems slightly positive. I feel a sense of anterior thoracic oppression, which changes with the movement of the body-trunk and increases while lying down. Pericarditis? I'm addled by questions, I'm worried about my family, and I'm isolated in the study. I wait until 6:30 then I call my friend and colleague Dr. P. at the hospital and announce that I'm driving to the emergency room (ER).

I park 6-700 $\mathrm{m}$ from the ER, it's a beautiful Sunday, the air is fresh, I walk, I feel strong but my head is light, I feel a bit skiddy. At the ER they are waiting for me, immediately they make me walk quickly for another $30 \mathrm{~m}$, the oxygen saturation $\left(\mathrm{SaO}_{2}\right)$ holds, always 98-99\%, heart rate (HR) $110 / \mathrm{min}$. The respiratory rate is normal $(16-17 / \mathrm{min})$. They accompany me to the COVID zone. They have all the necessary PPE but I recognize them, they greet me and make me feel at home. They drawn a blood gas analysis: $\mathrm{Ph} 7.51$, $\mathrm{PaO}_{2} 115 \mathrm{mmHg}, \mathrm{PCO}_{2} 27.5 \mathrm{mmHg}$. There is a respiratory alkalosis, but as you can see the capillary alveolus gradient is normal. "You must be a little anxious" they say to me. I'm not an anxious subject and I don't notice hyperventilating. Lactates $0.6 \mathrm{mmol} / \mathrm{L}$, normal bicarbonates $(24.5 \mathrm{mmol} / \mathrm{L})$, normal anion gap $(13.2 \mathrm{mEq} / \mathrm{L})$ excess of bases $0.1 \mathrm{mmol} / \mathrm{L}$. Therefore, it does not seem that respiratory alkalosis is a secondary disorder (to a metabolic acidosis) but, rather, that it is a primary disorder. Chest X-ray and lung ultrasound are normal, normal ECG. Due to my occupational exposure, I am swabbed twice, which is quite annoying. I' $m$ waiting for the other tests: $\mathrm{PCR}<5$, white blood cells $4520 / \mu \mathrm{L}$, lymphocytes and LDH normal. Creatinine and troponin are normal. I get home happy enough; maybe the swab is negative, hopefully, it is something else.

I try to rest, but I can't. In the afternoon I'm told that the swab is positive. It triggers quarantine for my family too. I get again the feeling of warmth in my face and upper body, headache and myalgia especially in the neck, the polyuria continues. $\mathrm{SaO}_{2} 98 \%$, HR 90-100 b/min. In the transition from cline to orthostatism, HR goes from 80-90 to 110-115/ min. It looks like a tachycardic response in orthostatic 
hypotension. I think the tachycardia is related to a state of dehydration due to polyuria, I force myself to drink more, but the more I drink the more the diuresis increases. I spend the night sleepless when I relax the frequency is reduced but the contractile strength of the heart increases and the heartbeat rumbles in the chest, especially if I put myself on my left side. It also seems to me that when I lie down I have more difficulty breathing, but saturation remains normal. After a while I'm lying down I have to get up to go to the bathroom to urinate, always polyuria. I go back to bed and I feel better, less heart palpitations, less dyspnea from clinostatism. Despite this, I try to sleep and every time I get to sleep there is something that wakes me up and prevents me from sleeping, it's like when you turn off a switch but immediately someone else from another room turns it back on. Did the sleep adjustment also go off?

The next day is similar, I force myself to drink, but it's useless, it only increases the polyuria. Moreover, if I drink more, the decubitus disorders, heart palpitations and dyspnea become more pronounced. I have to sleep with two pillows. I decide to drink less and I experience a clear reduction in orthopnea, even if my orthostatic tachycardia increases. It is as if in the decubitus, the venous return is extremely sensitive to the reduction or increase of the liquid intake, as well as the polyuria. I do some research and find a physiology article that demonstrates how hyperpnea, and not respiratory alkalosis, is the cause of polyuria [1]. In fact, deep ventilation causes a reduction in negative intrathoracic pressure that activates the low-pressure atrial and pulmonary vessels mechanoceptors, hence the production of natriuretic peptides and therefore polyuria. But why is there hyperpnea? Why is there tachycardia? I don't always have a high-temperature and it does not seem to me that I have pneumonia. I do a lung ultrasound (US) with a portable echo I have at home, there is a thickened and fragmented pleural line, especially in the upper anterior portions, in areas where I feel a sense of oppression, an anterior thoracic 'discomfort' (Fig. 1a). I look more carefully but I can't found complete B lines, there is no sign of interstitial disease. This fits with the normal capillary alveolar gradient. I do a heart US: sitting the chambers are normal, the vena cava is small and almost collapsed. I lie down and the vena cava increases in size $(22 \mathrm{~mm})$, even if it maintains a reduction in respiratory excursions. The left ventricle when lying down shows an increase in filling and contractile strength. Diastolic indices are normal and at most pseudonormal (Deceleration Time $180 \mathrm{~ms}$, with a HR around $90 \mathrm{~b} / \mathrm{min}$, I cannot make a tissue doppler). I am beginning to think that neural centers for the control of ventilation, volaemia, thermoregulation, waking-sleep rhythm, have lost their regulatory mechanisms, chasing balances that no longer appear possible.

Day 3. The fever disappears, by drinking less I have less orthopnea but I have to sleep with two pillows. I manage to get some sleep, 30-60 min at the most. A diffuse headache wakes up me. Now I have a slight cough. These complaints remind me of those I had when we conducted a study at 4,550 m above sea level, at the Regina Margherita Refuge Centre, on Monte Rosa [2]. There I had a saturation of 90-92\%, when I lay down I had the same feeling of being air-starved, and at wake up I had a dull headache, the sign of hypoxia during sleep. In point of fact, when lying down I desaturate (94-95\% saturation). I do a new lung US. In addition to the irregularities of the pleura, some $\mathrm{B}$ lines begin to appear (Fig. 1b, c), maximum 1-2 B lines per field without

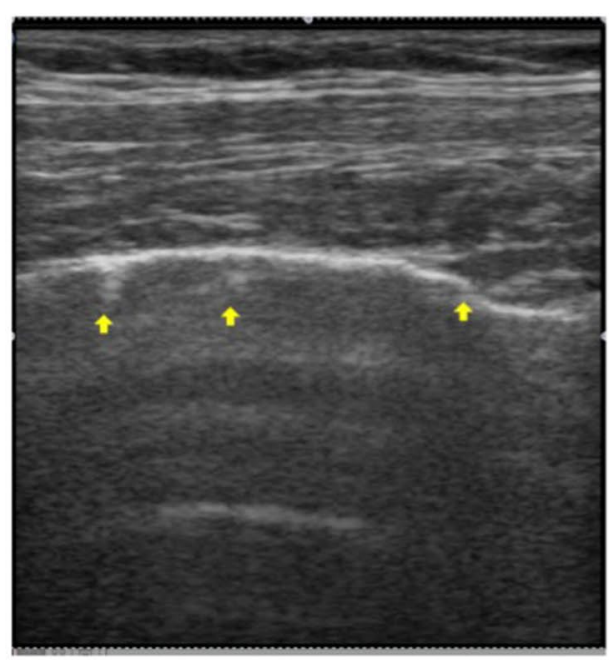

A

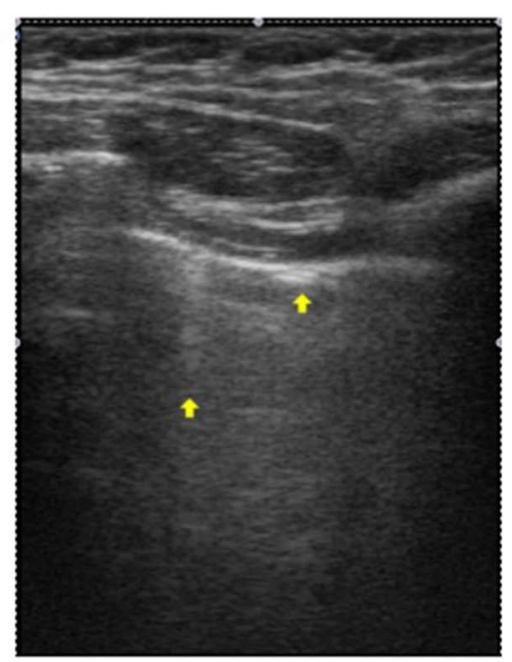

B

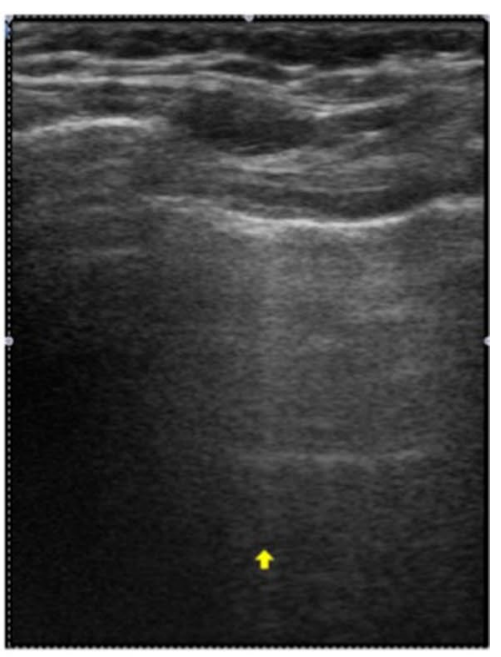

C

Fig. 1 a Thickened and fragmented pleural line, signs of initial pleural and subpleural disease. b Fragmented pleural line and incomplete formation of a B line (left arrow). c Complete B line 
definite pneumonia. Congestion? mild interstitial engagement? The echocardiogram has not changed. However, in case of doubt of myocardial damage, on my own (no one can come from the local health area), I repeat blood tests: normal PCR, no lymphopenia, normal procalcitonin and transaminases, troponin remains negative.

On the morning of the 4th day I start to have hypoosmia and hypogeusia. B. and E. (20 years old) tell me they've had it since the day before. During the day I start to move around but I become tachycardic when I go up and down the stairs. I do not desaturate during short time efforts. The pulmonary ultrasound picture remains similar. Since yesterday I no longer have fever. On the night of the 5th day, I can sleep more, at $2 \mathrm{~h}$ intervals, it seems to me that the polyuria is reduced. The coughing and the feeling of chest oppression persist. The pulmonary ultrasound picture is stationary. I think the pulmonary interstitial disease could evolve, so I start to take $200 \mathrm{mg}$ hydroxychloroquine morning and evening.

From the 6th day, I sometimes notice that at rest I have 96-97\%, then I move and my $\mathrm{SaO}_{2}$ goes up $98-99 \%$, it is as if now I have an input to breathless when I am at rest. Also B. and P. (24 years old), do not feel dyspnea but saturate 96-97\% at rest, on the ultrasound they have a pulmonary picture similar to mine. I worry that things may change and I start to administer hydroxychloroquine to them too.

Day 7, hyposmia and hypogeusia persist (all the others have it now too), the saturation behaviour remains more or less the same, my resting saturation remains 96-97\%. Polyuria is gone together with hyperpnea. We (me, E. and B.) notice that sometimes during the day, without being related to activity, heat appears on the face, we have a sensation of solar erythema, like a suntan (we have always been at home). Moreover, sleep has progressively increased, I finally sleep all night, and now I need to sleep even during the day. I associate the darker, tanned face to sleep, will there now be an overproduction of pro-oppiomelanocortin? Given the difficulty of running tests from home, I cannot verify this.

The 8th, 9th, and 10th days, we can gradually increase the activities in the house (cleaning, tidying up, I also go out into the garden a little bit) the boys now study and follow the lessons every day. I hope at this point that we are out of the tunnel. On the 14th day, I do a nasal swab: still positive. It will be negative only on day 42 .

We are now trying to get something useful out of all this, particularly looking at the early phase. I wish it could be useful for early diagnosis of COVID-19. Studying the course of the symptoms, supported by some observations on simple vital parameters, on the ultrasound of the lung and heart, and by simple blood tests (which always remained normal) I am more and more convinced that the virus has a particular tropism for brainstem centers of the autonomic nervous system. Experimental studies show that SARS-CoV viruses administered intranasally reach the hypothalamus, thalamus and brainstem through the olfactory nerves [3]. It is also interesting to read that Avian Influenza viral antigens were found in the nucleus of the solitary tract and the ambiguous nucleus $[4,5]$. The nucleus of the solitary tract processes information from mechanoreceptors in the lung vessels and atria and from chemoceptors in the lung and respiratory tract, while the efferent fibres provide innervation to smooth muscle cells, glands and blood vessels in the airways and lung [6]. It is not excluded that the efferent pathways are possibly involved in the propagation of the virus in the respiratory tract. From a clinical perspective, this dysfunction of the autonomic centers has a biphasic course. At the beginning there is an irritative phase: the first manifestation is hyperpnea (not polypnea, respiratory frequency remains normal), then polyuria (linked to hyperstimulation of mechanoreceptors of heart atria and large lung vessels), followed by tachycardia, insomnia, and a fever without inflammatory indices (central fever?). From the circulatory perspective, there is, on the one hand dehydration and tachycardia that is accentuated in orthostasis (some COVID-19 patients have syncopes from orthostatic hypotension) and, on the other, a venous pooling in intrathoracic veins during supine decubitus (similar to acute mountain sickness) that further stimulates polyuria. It is as if the venous vascular bed has become a static container, without vasomotility, completely dependent on its filling state: could it be a vasoparalysis or a dysregulation of vasomotor tone?

The initial irritative phase is followed, a few days later, by a phase characterized by a reduced activity of neural centers: no longer hyperpnea but a tendency to hypoventilation, hypoxia tolerance, and also hypersomnia, bradipsychism, relaxation. Significantly, there is a loss of taste and smell. This phase is also clinical important: precisely during the period in which the data in the literature tell us that one can turn towards interstitial pneumonia [7], and which I and some members of my family have perhaps only slightly developed, the ventilatory drive is reduced, with a consequent reduction in the sensation of dyspnea. It is an important phenomenon, to be aware of and know about, since it could make, not only the affected person but also whoever is assessing the subject (especially at a distance or indirectlyby phone, without objective evaluation and without oxymeter), underestimate the gravity of the situation. This could explain how many patients now arrive in the hospital with advanced stage pneumonia.

And while I study, research and try to understand, I think about this inanimate being (can we call it a tangle of RNA and proteins?). Inanimate yes, literally soulless, indifferent to our despair. And immediately I am reminded of the words of a great philosopher, Blaise Pascal, who says in Grandeur et misère de l'homme (Greatness and misery of man, thoughts $n^{\circ} 376$ and 377): 'In thought lies the greatness 
of man... a vapor, a drop of water are enough to kill him. But even if the universe crushed him, man would be more and more noble, because he knows he is dying, while the universe knows nothing about it... Through space the universe encompasses and swallows me up like a point, but through thought I comprehend the universe'. In French it sounds clearer and more incisive: 'Par l'espace, l'univers me comprend et m'engloutit comme un point; par la pensée, je le comprends'.

Acknowledgments We would like to thank Elizabeth Guerin from the University of Florence for her fundamental assistance in improving the manuscript form, concepts and language.

\section{Compliance with ethical standards}

Conflict of interest The authors declare that they have no conflict of interest.

Human and animal rights This article is the description of the clinical case of one of the authors and does not contain any studies with human particpants or animals performed by any of the authors.

Informed consent For this type of study, formal consent is not required.

\section{References}

1. Vidiendal Olsen N, Christensen H, Klausen T, Fogh-Andersen N, Plum I, Kanstrup IL, Hansen JM (1998) Effects of hyperventilation and hypocapnic/normocapnic hypoxemia on renal function and lithium clearance in humans. Anesthesiology 89(6):1389-1400 PubMed PMID: 9856713

2. Modesti PA, Vanni S, Morabito M, Modesti A, Marchetta M, Gamberi T, Sofi F, Savia G, Mancia G, Gensini GF, Parati G (2006) Role of endothelin-1 in exposure to high altitude: Acute Mountain Sickness and Endothelin-1 (ACME-1) study. Circulation 114(13):1410-1416 Epub 2006 Sep 18 PubMed PMID: 16982943

3. Xu J, Zhong S, Liu J et al (2005) Detection of severe acute respiratory syndrome coronavirus in the brain: potential role of the chemokine mig in pathogenesis. Clin Infect Dis 41:1089-1096

4. Li YC, Bai WZ, Hashikawa T (2020) The neuroinvasive potential of SARS-CoV2 may play a role in the respiratory failure of COVID-19 patients. J Med Virol. https://doi.org/10.1002/ jmv.25728[Epub ahead of print] Review

5. Matsuda K, Park CH, Sunden Y et al (2004) The vagus nerve is one route of transneural invasion for intranasally inoculated influenza a virus in mice. Vet Pathol 41:101-107

6. Kalia M, Mesulam MM (1980) Brain stem projections of sensory and motor components of the vagus complex in the cat: II. Laryngeal, tracheobronchial, pulmonary, cardiac, and gastrointestinal branches. J Comp Neurol 193:467-508

7. Wang D, Hu B, Hu C et al (2020) Clinical characteristics of 138 hospitalized patients with 2019 novel coronavirus-infected pneumonia in Wuhan China. JAMA. https://doi.org/10.1001/ jama.2020.1585

Publisher's Note Springer Nature remains neutral with regard to jurisdictional claims in published maps and institutional affiliations. 